DOI: $10.14529 / \operatorname{mmp} 170112$

\title{
THE CONTRIBUTION OF ANGELO FAVINI IN TWENTY YEARS OF JOINT RESEARCH (1996 - 2016)
}

\section{S. Romanelli, Universita' degli Studi di Bari Aldo Moro, Italy}

During his long activity in research, Angelo Favini has given several relevant contributions in many fields of mathematics. In our long collaboration, testified by more than thirty joint papers, I highly appreciated his deep competence, his brilliant intuitions, his extraordinary knowledge of the contemporary literature, in addition to a special acumen in finding possible critical points to be clarified, or better solved, during the preparation of the papers.

Here follows a short survey of the main problems studied in our joint papers over the last twenty years, often in collaboration with other outstanding mathematicians.

(1) At the beginning of 1990s there was a long standing open problem concerning the existence of analytic semigroups generated by second order elliptic differential operators degenerating at the boundary. It is well-known that the existence of a $\left(C_{0}\right)$ semigroup guarantees the wellposedness of the abstract Cauchy problem associated with the evolution equation governed by the generator, provided that the initial datum is in its domain. The analyticity of the semigroup provides, in addition, the best possible regularity of the solution, even by starting from the initial datum in the ambient space. As already known in Probability, or in Approximation Theory, in the space of continuous functions $C(\bar{\Omega})$ (here $\Omega$ is a bounded open subset of $\mathbb{R}^{N}$ with sufficiently smooth boundary $\partial \Omega$ ), a possibly degenerate on the boundary, second order elliptic operator $A$ can be naturally equipped with the so-called Wentzell boundary condition $A u=0$, introduced in the one-dimensional case by W. Feller in his pioneer work [7] (see also [6]), and in the multidimensional case by A.D. Wentzell [38]. For Wentzell boundary conditions in different spaces see e.g. [33,37]. Observe that, in an evolution equation $u_{t}=A u$, replacing $A u$ by $u_{t}$ in Wentzell boundary condition reveals that, under suitable regularity assumptions on the elements of the domain of $A$, a solution $u$ of the equation with Wentzell boundary condition remains constant with respect to $t$ along the boundary. An easy example of degenerate elliptic second order differential operator on the space $C[0,1]$ equipped with Wentzell boundary conditions is $A_{j} u=x^{j}(1-x)^{j} u^{\prime \prime}, j>0$, well-known also for governing some evolution problems in genetics. In [1] the existence of analytic semigroups generated by $A_{j}$ was proved for $j \geq 2$ on $C[0,1]$ by using different types of boundary conditions, including Wentzell's ones. After that, much attention was paid to this problem and relevant contributions by A. Favini et al. appeared in different papers, where, in addition to the space of continuous functions, as in [27,30,31], also $L^{p}$ spaces, with or without weights, and Sobolev spaces were considered, see e.g. [2,20,28,29]. Results concerning the wave equation with Wentzell boundary conditions in $C[0,1]$ were also obtained in [16].

(2) In the real-valued space $C(\bar{\Omega})$ other interesting problems concern the existence of Feller semigroups generated by second-order elliptic differential operators degenerating on $\partial \Omega$ and equipped with different boundary conditions, including Wentzell's ones. In this framework, some enlightening results were obtained by K. Taira, A. Favini and S. Romanelli in the papers [34-36], under suitable regularity assumptions on the coefficients of the operator and on $\partial \Omega$. 
(3) If $A$ denotes a second order, linear or nonlinear, elliptic differential operator, a general boundary condition including both Robin (and Dirichlet, Neumann) boundary conditions $b \frac{\partial u}{\partial n}+c u=0$, relevant for $L^{p}$ spaces, and Wentzell boundary conditions $A u=0$, relevant for spaces of continuous functions, arises naturally as general Wentzell boundary condition given by

$$
(G W B C) \quad a A u(x)+b \frac{\partial u(x)}{\partial n}+c u(x)=0, \quad x \in \partial \Omega,
$$

where $(a, b, c) \neq(0,0,0)$ and $\frac{\partial u}{\partial n}$ is the outer normal derivative of $u$. In [13] A. Favini, G.R. Goldstein, J.A. Goldstein and S. Romanelli introduced $(G W B C$ ) for the operator $A u=\alpha u^{\prime \prime}$ with domain

$$
D(A)=\left\{u \in C[0,1] \cap C^{2}(0,1): A u \in C[0,1], u \text { satisfies }(G W B C)\right\}
$$

in $C[0,1]$. Note that, in this case, $(G W B C)$ consists of two conditions and reads as

$$
a_{j} A u(j)+(-1)^{j} b_{j} u^{\prime}(j)+c_{j} u(j)=0, \quad j=0,1,
$$

with $a_{j}, b_{j}, c_{j}$ real numbers.

Therein, under the assumptions that $\alpha \in C(0,1), \alpha>0$ and $\frac{1}{\alpha} \in L^{1}(0,1)$ ( $\alpha$ can vanish at $\{0,1\}$ ) and suitable assumptions on $a_{j}, b_{j}, c_{j}, j=0,1$, the authors stated sufficient conditions yielding meaningful properties of the operator $A$ in $C[0,1]$, as density of the domain, closedness, dissipativity and range condition. For instance, $A$ is densely defined and $m$-dissipative whenever $a_{0}=1=a_{1}$ and $(-1)^{j} b_{j} \leq 0$ and $c_{j} \geq 0$, with $c_{j}=0$ if $b_{j}=0, j=0,1$. More general nonlinear operators and nonlinear $(G W B C)$ can also be considered (see e.g. [17]). Hence, the previous results provide extremely useful tools in the study of wellposedness for abstract Cauchy problems associated with a wide class of evolution problems.

(4) The wellposedness of abstract Cauchy problems with $(G W B C)$ in $L^{p}$ spaces, $1 \leq$ $p<\infty$ requires the introduction of special spaces, as, for instance, discussed in the simple case of $A u=u^{\prime \prime}$, acting on $L^{2}(0,1)$, equipped with $(G W B C)$, see [18, Section 5]. Hence, in order to study $\left(C_{0}\right)$-semigroups governed by operators equipped with $(G W B C)$ in spaces of $L^{p}$-type, a new approach is necessary. In [18] A. Favini, J.A. Goldstein, G.R. Goldstein and S. Romanelli assumed that $A$ is the elliptic second order differential operator in divergence form $A u=\nabla \cdot(a \nabla)$, where $a \in C^{1}(\bar{\Omega}), a$ is nonnegative and satisfies $\Gamma:=\{x \in \partial \Omega$ : $a(x)>0\} \neq \emptyset$, and $A$ is equipped with the following ( $G W B C$ )

$$
A u+\beta \frac{\partial u}{\partial n}+\gamma u=0 \quad \text { on } \quad \Gamma
$$

with $\beta, \gamma$ nonnegative functions in $C^{1}(\partial \Omega), \beta>0$. Note that, if we consider the evolution equation $u_{t}=A u$ equipped with $(G W B C)$, under assumptions of sufficient regularity for $u$, then we can plug the term $u_{t}=A u$ in the boundary condition and obtain the condition $u_{t}+\beta \frac{\partial u}{\partial n}+\gamma u=0$. Hence, the term $A u$ corresponds to introduce a dynamic condition on the boundary. Coming back to the introduction of the new spaces, let us assume that $\Gamma=\partial \Omega$. For $1 \leq p<\infty$, the correct $L^{p}$ space to consider is $X_{p}:=L^{p}(\bar{\Omega}, d \mu)$, where $d \mu=\left.\left.d x\right|_{\Omega} \oplus \frac{a d S}{\beta}\right|_{\partial \Omega}$. Here $d x$ denotes the Lebesgue measure on $\Omega$ and $\frac{a d S}{\beta}$ denotes the natural surface measure $d S$ on $\partial \Omega$ with weight $\frac{a}{\beta}$. For $p=\infty$ the space $X_{\infty}$ can 
be identified with $C(\bar{\Omega})$. One can interpret $X_{p}, 1 \leq p<\infty$, as the completion of $C(\bar{\Omega})$ with respect to the norm $\|\cdot\|_{X_{p}}$ given by $\|U\|_{X_{p}}=\left(\int_{\Omega}|u|^{p} d x+\int_{\partial \Omega}|u|^{p} \frac{a d S}{\beta}\right)^{\frac{1}{p}}$, where, if $u \in C(\bar{\Omega})$, we consider $U=\left(\left.u\right|_{\Omega},\left.u\right|_{\partial \Omega}\right)$. In general, a member of $X_{p}$ is a pair $H=(f, g)$, where $f \in L^{p}(\Omega, d x)$ and $g \in L^{p}\left(\partial \Omega, \frac{a d S}{\beta}\right)$ and, for $p<\infty, f$ may not have a trace on $\partial \Omega$, and even if $f$ does, this trace needs not equal $g$. For $p=2, X_{2}$ is a Hilbert space equipped with the inner product $<H_{1}, H_{2}>_{X_{2}}=<f_{1}, f_{2}>_{L^{2}(\Omega)}+<g_{1}, g_{2}>_{L^{2}\left(\partial \Omega, \frac{a d S}{\beta}\right)}$, with $H_{i}=\left(f_{i}, g_{i}\right) \in X_{2}, i=1,2$. Under previous assumptions, in [18], the authors proved that there exists a $\left(C_{0}\right)$ semigroup generated by the closure of the realization of $A$ in $X_{p}$, $1 \leq p \leq \infty$, and this semigroup is analytic if $1<p<\infty$. In addition, $A$ is essentially self-adjoint on $X_{2}$. Existence and analyticity results for the semigroup associated with $A$ remain true also for more general elliptic versions of $A$, equipped with the corresponding $(G W B C)$. See [11]. It is very important to point out that there is a precise physical derivation of $(G W B C)$, as it was shown in details by G.R. Goldstein in [32]. For instance, in the case of the heat equation, in the evaluation of the total heat content of the region, the use of $(G W B C)$ allows to consider also the contribution of a heat source located on the boundary, while in all usual approaches concerning the traditional boundary conditions, this type of contribution appears completely neglected. All the above results opened the way to a more and more wide literature and to a diffused interest of the international community of mathematicians, including those ones involved in the study of dynamical boundary conditions. Thus, in my opinion, the above results represent the milestone of our scientific collaboration.

(5) In the last years, starting from the paper [11], A. Favini, G.R. Goldstein, J.A. Goldstein, E. Obrecht and S. Romanelli found a more complete formulation of $(G W B C)$ in this form

$$
(G G W B C) \quad A u+\beta \frac{a \partial u}{\partial n}+\gamma u-q \beta \Delta_{L B} u=0, \quad \text { on } \quad \partial \Omega
$$

including the term with the Laplace - Beltrami operator $\Delta_{L B}$ having nonnegative constant coefficient $q$. In $[11,12]$ the authors assumed that $A$ is a uniformly elliptic operator in divergence form of general type. Here we refer to $A$ given as in (4) and to the assumptions on the coefficients in $(G G W B C)$ as before. Then the realization of $A$ in $X_{2}$, with a suitable domain, including $(G G W B C)$, has a dissipative self-adjoint closure which generates an analytic semigroup in $X_{2}$ with angle of analyticity $\frac{\pi}{2}$. In the same papers [11,12], under $C^{\infty}$ regularity assumptions on $\partial \Omega$, on all the coefficients of $A$ and on $\beta, \gamma$, we also showed that the closure of the realization of $A$ in $X_{p}, 1<p<\infty$, is $m$-dissipative and generates an analytic semigroup having sector of analyticity depending on the moduli of ellipticity of $A$. Moreover, its domain can be explicitly described. In a long series of subsequent papers by A. Favini, G.R. Goldstein, J.A. Goldstein, S. Romanelli and other coauthors, as G.M. Coclite, C.G. Gal and E. Obrecht, see e.g. [3-5, 8, 12, 21, 25], additional interesting results were obtained with respect to continuous dependence of the solutions from the boundary coefficients, hyperbolic problems, nonautonomous wave equation, nonlinear operators, nonsymmetric operators, unbounded domains.

(6) Extensions of some previous results described in (5) can be obtained also in the case of some classes of higher order operators acting on $X_{2}$. See e.g. [22, 24]. In these papers Wentzell boundary conditions together with lower order boundary conditions are 
associated with this type of operators. For instance, in $X_{2}=L^{2}(\Omega, d x) \oplus L^{2}\left(\partial \Omega, \frac{d S}{\beta}\right)$, a fourth order operator of the type $A u=\Delta(a \Delta) u$, equipped with both boundary conditions $A u+\beta \frac{\partial(a \Delta u)}{\partial n}+\gamma u=0$ and $\Delta u=0$ is essentially self-adjoint and bounded below, provided that $a \in C^{4}(\bar{\Omega}), a>0, \partial \Omega$ is $C^{4}$ and $\beta, \gamma \in C^{3+\epsilon}(\partial \Omega)$. A more complete classification of $(G W B C)$ for the fourth order differential operator $A u=u^{\prime \prime \prime \prime}$ acting on $X_{2}$, with $\Omega=(0,1)$, can be found in [23] where we obtained a characterization of the symmetry and sufficient conditions for self-adjointness and quasi- $m$-accretivity of $A$.

\section{References}

1. Attalienti A., Romanelli S. On Some Classes of Analytic Semigroups on $C([a, b])$ Related to $\mathbb{R}$ or $\Gamma$-Admissible Mappings. Evolution Equations, N.Y., M. Dekker, 1995, p. 29-34.

2. Barbu V., Favini A., Romanelli S. Degenerate Evolution Equations and Regularity of Their Associated Semigroups. Funkcialaj Ekvacioj, 1996, vol. 39, pp. 421-448.

3. Coclite G.M., Favini A., Gal C.G., Goldstein G.R., Goldstein J.A., Obrecht E., Romanelli S. The Role of Wentzell Boundary Conditions in Linear and Nonlinear Analysis. Advances in Nonlinear Analysis: Theory, Methods and Applications. 2009, vol. 3, pp. 279-292.

4. Coclite G.M., Favini A., Goldstein G.R., Goldstein J.A., Romanelli S. Continuous Dependence on the Boundary Conditions for the Wentzell Laplacian. Semigroup Forum, 2008, vol. 77, pp. 101-108. DOI: 10.1007/s00233-008-9068-2

5. Coclite G.M., Favini A., Goldstein G.R., Goldstein J.A., Romanelli S. Continuous Dependence in Hyperbolic Problems with Wentzell Boundary Conditions. Communications on Pure and Applied Analysis, 2014, vol. 13, pp. 419-433. DOI: 10.3934/cpaa.2014.13.419

6. Clement Ph., Timmermans C. On $C_{0}$-Semigroups Generated by Differential Operators Satisfying Ventcel's Boundary Conditions. Indagationes Mathematicae, 1986, vol. 89, pp. 379-387. DOI: 10.1016/1385-7258(86)90023-5

7. Feller W. The Parabolic Differential Equations and the Associated Semigroups of Transformations. Annals of Mathematics, 1952, vol. 55, no. 3, pp. 468-519. DOI: $10.2307 / 1969644$

8. Favini A., Gal C.G., Goldstein G.R., Goldstein J.A., Romanelli S. The Non-Autonomous Wave Equation with General Wentzell Boundary Conditions. Proceedings of the Royal Society of Edinburgh. Section A. Mathematics, 2005, vol. 135 A, pp. 317-329.

9. Favini A., Goldstein G.R., Goldstein J.A., Obrecht E., Romanelli S. The Laplacian with Generalized Wentzell Boundary Conditions. Evolution Equations: Applications to Physics, Industry, Life Sciences and Economics, Birkhäuser Verlag, 2003, vol. 55, pp. 169-180. DOI: $10.1007 / 978-3-0348-8085-5 \_13$

10. Favini A., Goldstein G.R., Goldstein J.A., Obrecht E., Romanelli S. General Wentzell Boundary Conditions and Analytic Semigroups on $W^{1, p}(0,1)$. Applicable Analysis, 2003, vol. 82, issue 9, pp. 927-935. DOI: 10.1080/00036810310001596601

11. Favini A., Goldstein G.R., Goldstein J.A., Obrecht E., Romanelli S. Elliptic Operators with General Wentzell Boundary Conditions, Analytic Semigroups and the Angle Concavity Theorem. Mathematische Nachrichten, 2010, vol. 283, pp. 1-18. DOI: $10.1002 /$ mana.200910086

12. Favini A., Goldstein G.R., Goldstein J.A., Obrecht E., Romanelli S. Nonsymmetric Elliptic Operators with Wentzell Boundary Conditions in General Domains. Communications on Pure and Applied Analysis, 2016, vol. 15, pp. 2475-2487. DOI: 10.3934/cpaa.2016045 
13. Favini A., Goldstein G.R., Goldstein J.A., Romanelli S. $C_{0}$-Semigroups Generated by Second Order Differential Operators with General Wentzell Boundary Conditions. Proceedings of the American Mathematical Society, 2000, vol. 128, pp. 1981-1989. DOI: 10.1090/S0002-9939-0005486-1

14. Favini A., Goldstein G.R., Goldstein J.A., Romanelli S. Generalized Wentzell Boundary Conditions and Analytic Semigroups in $C[0,1]$. Semigroups of Operators: Theory and Applications, Birkhäuser, Basel, 2000, pp. 125-130.

15. Favini A., Goldstein G.R., Goldstein J.A., Romanelli S. On Some Classes of Differential Operators Generating Analytic Semigroups. Evolution Equations and Their Applications in Physical and Life Sciences. N.Y., Marcel Dekker, 2000, pp. 105-120

16. Favini A., Goldstein G.R., Goldstein J.A., Romanelli S. The One Dimensional Wave Equation with Wentzell Boundary Conditions. Differential Equations and Control Theory. N.Y., Marcel Dekker, 2001, pp. 139-145.

17. Favini A., Goldstein G.R., Goldstein J.A., Romanelli S. Nonlinear Boundary Conditions for Nonlinear Second Order Differential Operators on $C[0,1]$. Archiv der Mathematik, 2001, vol. 76, pp. 391-400. DOI: $10.1007 /$ PL00000449

18. Favini A., Goldstein G.R., Goldstein J.A., Romanelli S. The Heat Equation with Generalized Wentzell Boundary Conditions. Journal of Evolution Equations, 2002, vol. 2, pp. 1-19. DOI: $10.1007 / \mathrm{s} 00028-002-8077-\mathrm{y}$

19. Favini A., Goldstein G.R., Goldstein J.A., Romanelli S. Degenerate Second Order Differential Operators Generating Analytic Semigroups in $L^{p}$ and $W^{1, p}$. Mathematische Nachrichten, 2002, vol. 238, pp. 78-104. DOI: 10.1002/1522-2616(200205)238:1<78::AIDMANA78 $>3.0 . \mathrm{CO} ; 2-\mathrm{X}$

20. Favini A., Goldstein G.R., Goldstein J.A., Romanelli S. General Wentzell Boundary Conditions, Differential Operators and Analytic Semigroups in $C[0,1]$. Boletim da sociedade paranaense de matematica, 2002, vol. 20, no. 1-2, pp. 93-102.

21. Favini A., Goldstein G.R., Goldstein J.A., Romanelli S. The Heat Equation with Nonlinear General Wentzell Boundary Condition. Advances in Differential Equations, 2006, vol. 11, pp. $481-510$.

22. Favini A., Goldstein G.R., Goldstein J.A., Romanelli S. Fourth Order Ordinary Differential Operators with General Wentzell Boundary Conditions. Differential Equations: Direct and Inverse Problems. Boca Raton, Chapman \& Hall/CRC, 2006, vol. 251, pp. 61-74.

23. Favini A., Goldstein G.R., Goldstein J.A., Romanelli S. Classification of General Wentzell Boundary Conditions for Fourth Order Operators in One Space Dimension. Journal of Mathematical Analysis and Applications, 2007, vol. 333, pp. 219-235. DOI: $10.1016 /$ j.jmaa.2006.11.058

24. Favini A., Goldstein G.R., Goldstein J.A., Romanelli S. Fourth Order Operators with General Wentzell Boundary Conditions. Rocky Mountain Journal of Mathematics, 2008, vol. 38, pp. 445-460. DOI: 10.1216/RMJ-2008-38-2-445

25. Favini A., Goldstein G.R., Goldstein J.A., Romanelli S. Wentzell Boundary Conditions in the Nonsymmetric Case. Mathematical Modelling of Natural Phenomena, 2008, vol. 3, pp. 143-147. DOI: $10.1051 / \mathrm{mmnp}: 2008046$

26. Favini A., Goldstein G.R., Goldstein J.A., Romanelli S. Selfadjointness of Degenerate Elliptic Operators on Higher Order Sobolev Spaces. Discrete and Continuous Dynamical Systems. Series S, 2011, vol. 4, pp. 581-593.

27. Favini A., Goldstein G.R., Goldstein J.A., Romanelli S. Analytic Feller Semigroups via Hypergeometric Series. African Diaspora Journal of Mathematics, 2014, vol. 17, pp. 1-9. 
28. Favini A., Goldstein J.A., Romanelli S. An Analytic Semigroup Associated to a Degenerate Evolution Equation. Stochastic Processes and Functional Analysis, N.Y., Marcel Dekker, 1997, vol. 186, pp. 85-100.

29. Favini A., Goldstein J.A., Romanelli S. Analytic Semigroups on $L_{w}^{p}(0,1)$ and on $L^{p}(0,1)$ Generated by Some Classes of Second Order Differential Operators. Taiwanese Journal of Mathematics, 1999, vol. 3, pp. 181-210.

30. Favini A., Romanelli S. Degenerate Second Order Operators as Generators of Analytic Semigroups on $C[0,+\infty]$ or on $L_{\alpha^{-\frac{1}{2}}}^{p}(0,+\infty)$. Approximation and Optimization. Proceedings of the International Conference on Approximation and Optimization. Vol. II, 1997, pp. 93-100.

31. Favini A., Romanelli S. Analytic Semigroups on $C[0,1]$ Generated by Some Classes of Second Order Differential Operators. Semigroup Forum, 1998, vol. 56, pp. 362-372.

32. Goldstein G.R. Derivation and Physical Interpretation of General Boundary Conditions. Advances in Differential Equations, 2006, vol. 11, no. 4, pp. 457-480.

33. Goldstein J.A., Lin C.Y. Highly Degenerate Parabolic Boundary Value Problems. Differential and Integral Equations, 1989, vol. 2, no. 2, pp. 216-227.

34. Romanelli S., Favini A., Goldstein G.R., Goldstein J.A., Taira K. Degenerate Elliptic Operators with General Boundary Conditions and Feller Semigroups. Far East Journal of Applied Mathematics, 2006, vol. 24, pp. 1-22.

35. Taira K., Favini A., Romanelli S. Feller Semigroups Generated by Degenerate Elliptic Operators. Semigroup Forum, 2000, vol. 60, pp. 296-309. DOI: 10.1007/s002339910022

36. Taira K., Favini A., Romanelli S. Feller Semigroups and Degenerate Elliptic Operators with Ventcel' Boundary Conditions. Studia Mathematica, 2001, vol. 145, no. 1, pp. 17-53. DOI: $10.4064 / \mathrm{sm} 145-1-2$

37. Vespri V. Analytic Semigroups, Degenerate Elliptic Operators and Applications to Nonlinear Cauchy Problems. Annali di Matematica Pura ed Applicata, 1989, vol. 155, issue 1, pp. 353-388. DOI: $10.1007 /$ bf01765950

38. Wentzell A.D. On Boundary Conditions for Multi-Dimensional Diffusion Processes. Theory of Probability and Its Applications, 1959, vol. 4, no. 2, pp. 164-177. DOI:10.1137/1104014

Received January 17, 2017 